\title{
ADDITIVE MANUFACTURING OF HOLLOW MICRONEEDLES FOR INSULIN DELIVERY
}

\author{
Iakovos Xenikakis ${ }^{1}$, Konstantinos Tsongas ${ }^{2}$, Emmanouil K Tzimtzimis ${ }^{2}$, \\ Dimitrios Tzetzis $^{2}$, Dimitrios Fatouros ${ }^{1}$ \\ ${ }^{1}$ Laboratory of Pharmaceutical Technology, Department of Pharmacy, School of Health Sciences, Aristotle University of \\ Thessaloniki, Thessaloniki GR-54124, Greece \\ ${ }^{2}$ Digital Manufacturing and Materials Characterization Laboratory, School of Science and Technology, International \\ Hellenic University, 14km Thessaloniki - N. Moudania, 57001, Greece
}

Corresponding author: Iakovos Xenikakis, iakpolxen@pharm.auth.gr

\begin{abstract}
Microneedles (MN) are miniature devices capable of perforating painlessly stratum corneum and delivering active ingredients in the inner epidermal layers. Hollow microneedles (HMNs) are highly detailed objects due to their internal microchannels and thus, their fabrication with Additive Manufacturing (AM) is a challenging task. Vat polymerization techniques provide a sufficient accuracy for such microstructures. Differentiated from other approaches where stereolithography and 2-photon polymerization were adopted, this paper presents the 3D-printing of HMNs purposed for insulin delivery, using the more economic Liquid Crystal Display (LCD) method. First, different geometries (hexagon, square pyramid, beveled) were 3D printed with constant height and varying curing time, printing angle and layer resolution. Quality features in each case were captured with optical and scanning electron microscopy (SEM). The most promising geometry was found to be the beveled one due to the more refined tip area and the feasibility of non-clogged microchannel formation. Among printing parameters, printing angle proved to be the most influential, as it affects resin flow phenomenon during printing process. Lastly, optimized HMN geometry was the beveled configuration, where the average height was measured $900 \mu \mathrm{m}, 3 \mathrm{D}$ printing angle was set at $-45^{\circ}$, the curing time was $10 \mathrm{~s}$ per layer and the optimal layer height was $30 \mu \mathrm{m}$.
\end{abstract}

Key words: hollow microneedles, liquid crystal display, vat polymerization, printing angle, beveled geometry, insulin, transdermal delivery.

\section{INTRODUCTION}

Needle phobia is often responsible for avoidance of medical treatment with severe health consequences $[1,2]$. Especially in cases like diabetes Type I or II where insulin uptake is part of the daily routine, serious concerns about patients' compliance are posed [3]. As insulin is a protein based drug and thus prone to degradation in gastrointestinal tract, subcutaneous administration is the only appropriate route of administration causing much of pain and inconvenience [4]. Hollow microneedles (HMNs), as devices possessing minimal dimensions, have the potential to replace the fear inducing syringe based administration alleviating everyday life of millions of patients [5]. HMNs have been primarily produced by microelectromechanical methods [6]. These methods are complicate procedures conducted with the aid of sophisticated machinery, and require lots of working hours for their completion. In contrast, Additive Manufacturing (AM) offers a much more convenient way of manufacturing due to the following reasons: a) HMNs are first designed with Computer Aided Design (CAD) software and designs can be saved as .stl files and be modified if necessary, b) manufacture is a single step procedure in which layers of the printing material are successively deposited on a building platform with no use of extra tooling and c) machinery required (computer and 3D-printer) is quite simple, which in fact can result in establishing small production units all over the world including developing countries [7]. HMNs have been produced mainly by means of vat polymerization methods (VPM) namely stereolithography (SLA) [8-10] and 2-photon polymerization (2-PP) [11-15]. Other methods include Fused Filament Fabrication (FFF) [16,17], and Selective Laser Micro Melting (SL $\mu \mathrm{M})$ [18]. SLA and 2-PP, like most VPMs, promise high accuracy between designs and printlets. Yet, their main disadvantage is that production costs are high, and as a consequence logistics are increased mitigating the possibility of global commercialization. Among VPMs, liquid crystal display (LCD) holds promise as it has a satisfying accuracy (below $50 \mu \mathrm{m}$ ) with low costs. Hence, LCD method was opted for HMN fabrication with the 
purpose of further utilizing them for insulin delivery. First, three geometries were designed using a CAD software with constant height. Other parameters like printing angle, layer height and curing time were adjusted using a slicing software and varied to find out the optimal printing values. The printing material was a biocompatible resin used for dentistry applications. Finally, optical microscopy and scanning electron microscopy (SEM) were used to measure geometrical characteristics and capture quality features of 3Dprinted HMNs.

\section{EXPERIMENTAL}

A variety of CAD geometries were designed namely square pyramid, beveled and hexagon, as shown in Figure 1. Beveled configuration results after crosssectioning of a hollow cylinder under a specific angle. Their height had a constant value of $1000 \mu \mathrm{m}$ for all designs, whereas tip area was less than
$4000 \mu \mathrm{m}^{2}$. Microchannel projection diameter and interspacing was $500 \mu \mathrm{m}$ and $3000 \mu \mathrm{m}$ respectively. Patches were $3 \times 3 \mathrm{HMNs}$ arrays of $225 \mathrm{~mm}^{2}$ square surface (15mm x 15mm).

A Phrozen Shuffle 2018 3D-printer utilizing a $2 \mathrm{~K}$ LED screen was employed. 3D printing material was the biocompatible Class IIa resin NextDent Ortho Rigid (NextDent, Netherlands). The final parameters were adjusted in ChiTuBox 64 slicing software. Supports were carefully added far from critical features (microchannel and HMN corpus). Layer height was set at $10-30 \mu \mathrm{m}$ range. Lifting speed was $400 \mu \mathrm{m} / \mathrm{s}$. Each $3 \mathrm{D}$ printing cycle included 10s curing time for each layer except of the first 12 layers (burn in layers) whose curing time was 70 s to ensure printlet attachment on building platform. Curing time of $5 \mathrm{~s}$ and $15 \mathrm{~s}$ were also attempted. Four $3 \mathrm{D}$ printing angles were tested: $0^{\circ}$, $+45^{\circ}, 90^{\circ}-45^{\circ}$. The $3 \mathrm{D}$ printing process had a minimum duration of $3 \mathrm{~h}$ and $30 \mathrm{~min}$.

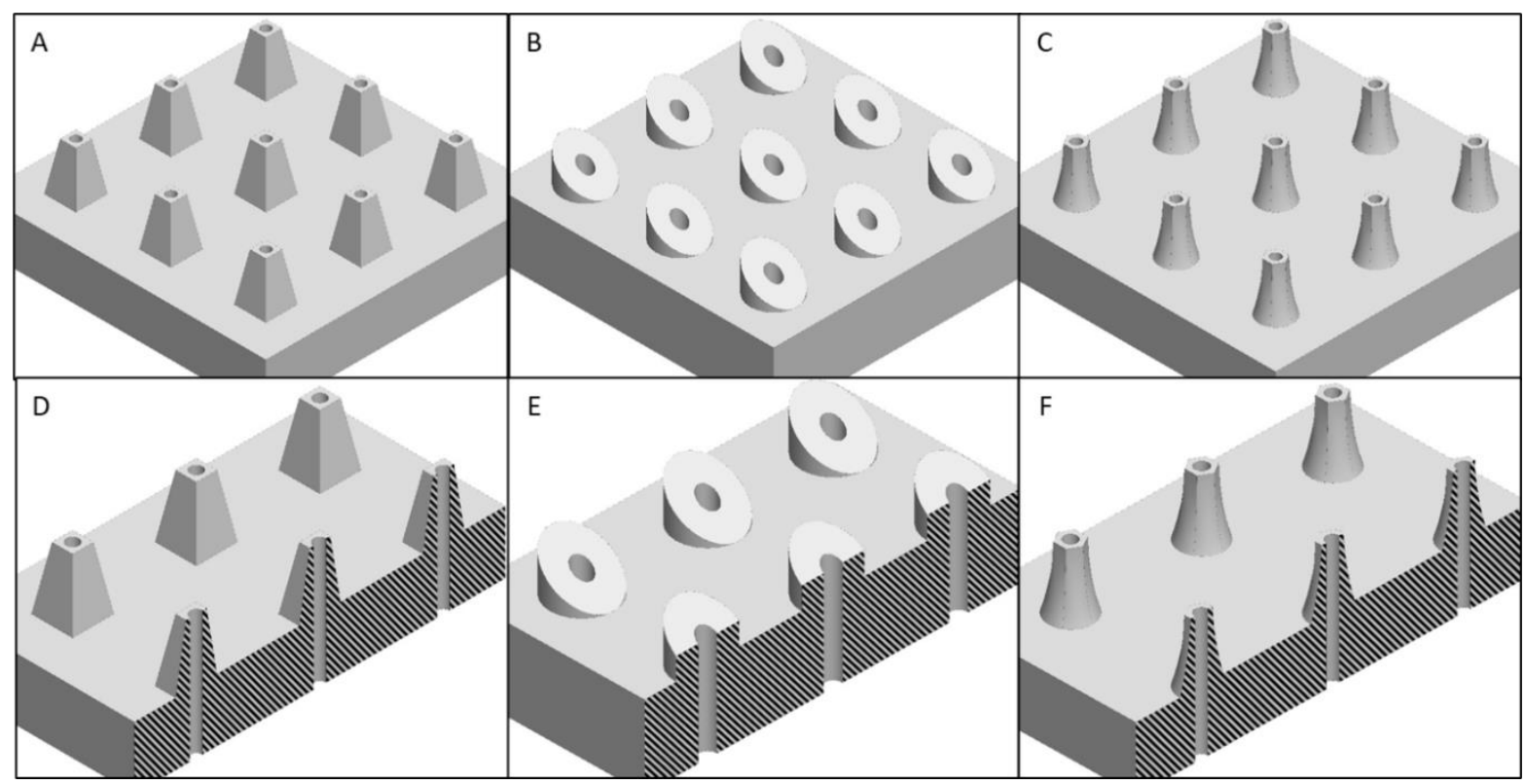

Fig. 1. (A)-(C): CAD designs of square pyramid, beveled and hexagon HMNs. (D)-(F):

The corresponding cross-sections

At the end of the process, HMNs were immersed in isopropyl-alcohol for $5 \mathrm{~min}$ under ultrasonic bath (Hielscher UP200s, Teltow, Germany) to remove any unsolidified resin residuals. Finally, HMNs were dried in an air-circulated oven (Binder FD56, Tuttlinger, Germany) for $20 \mathrm{~min}$ at $30^{\circ} \mathrm{C}$. Supports were carefully cut using scalpels. Fabricated HMNs were inspected and measured for their actual dimensions using the optical microscope DinoLite AD7013MZT (AnMo Electronics, Hsinchu, Taiwan) and the related software DinoLite 2.0. Scanning Electron Microscopy (SEM) was also implemented (Phenom ProX, ThermoFischer, USA). HMNs were attached on a metal stub using double-sided adhesive tape and inserted in charge reducing sample holder. The whole assembly was lastly inserted in SEM microscope under vacuum.

\section{RESULTS AND DISCUSSION}

Among the geometries tested, the beveled configuration was the only candidate that resulted in HMN fabrication with non-clogged microchannels, as shown in Figures 2 (a)-(h). For the rest geometries, no experimental conditions could yield in a similar result. Referring to beveled microneedles, under $0^{\circ},+45^{\circ}, 90^{\circ} 3 \mathrm{D}$ printing angles no hollow microchannel formation was observed. $-45^{\circ}$ was the only occasion where 


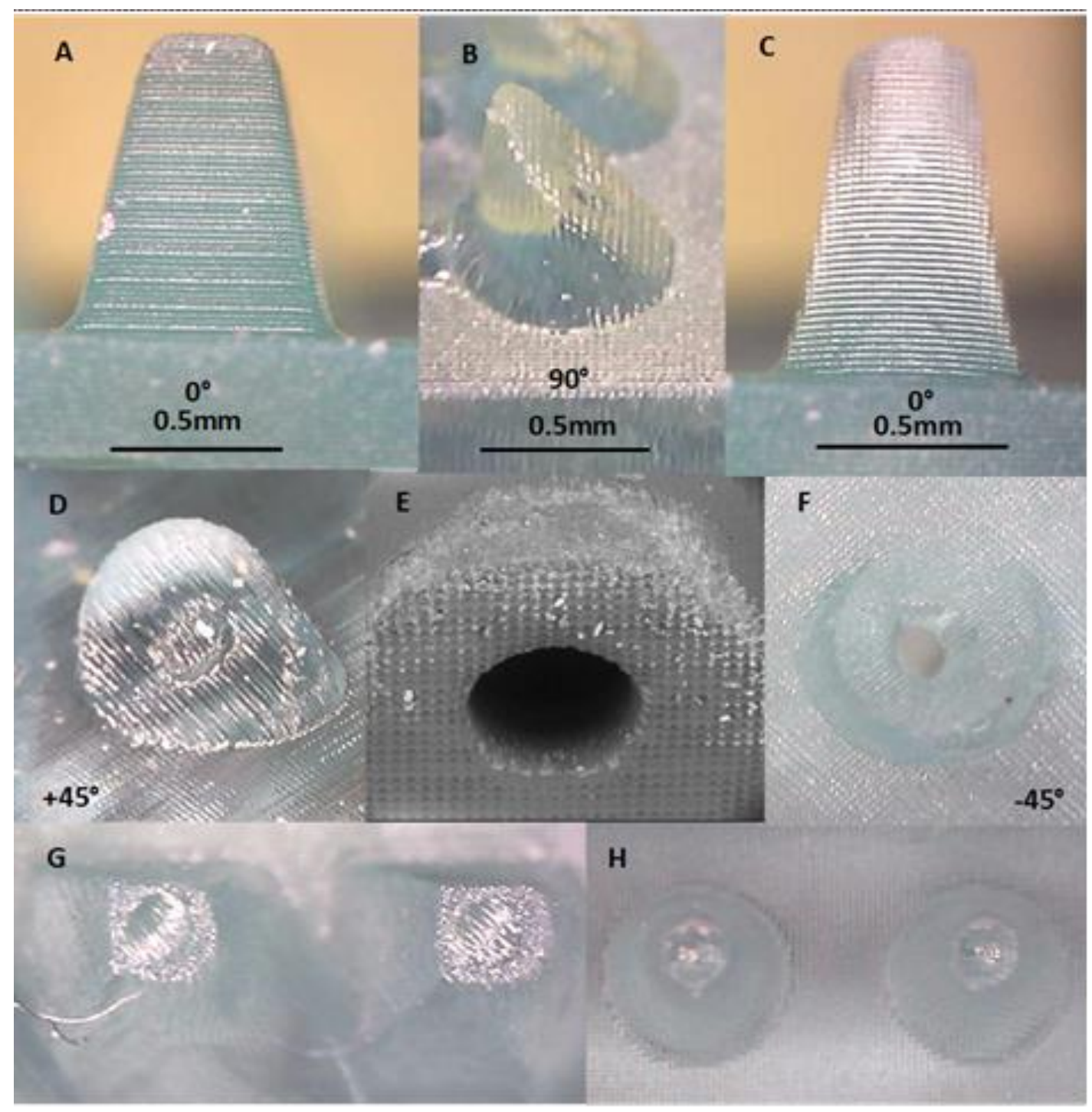

Fig. 2. 3D printed microneedles (a) at $0^{\circ}$ with square pyramid, (b) at $90^{\circ}$ with beveled and (c) at $0^{\circ}$ with hexagon configuration. (d) Beveled geometry at $+45^{\circ}$, (e) SEM image of a beveled HMN, (f) Beveled geometry at $-45^{\circ}$. (g),

(h) Top views of square pyramid and hexagon microneedles, respectively

HMNs were successfully 3D printed reaching $100 \%$ microchannel formation (9 out of 9 ). The use of $5 \mathrm{~s}$ curing time resulted in inadequate polymerization, whereas $15 \mathrm{~s}$ increased printing time with no evident result. Layer resolution of $10 \mu \mathrm{m}$ was too low for the printer's capability and printings failed, whereas $20 \mu \mathrm{m}$ and $30 \mu \mathrm{m}$ resulted in successful printings with no significant differences between each other in quality features.

Tip region was too coarse for hexagon and square pyramid microneedles and the corresponding tip area was high $\left(>10.000 \mu \mathrm{m}^{2}\right)$. Beveled HMNs had in general the most refined tip area, which was closer to the original design (around $4500 \mu \mathrm{m}^{2}$ ). It was also observed that $-45^{\circ}$ gave slightly larger tip areas, but it was the only printing angle resulting in HMN formation. Microneedle length was shorter than the designed by $5 \%$. This phenomenon was more pronounced for beveled geometry $(10 \%)$. As such, beveled HMNs had an average length of
$900 \mu \mathrm{m}$. Microchannel projection diameter was $500 \mu \mathrm{m}$.

Deviations between designs and 3D printed objects are common in VPMs. It has been reported elsewhere that the measured height is closer to the designed one, as the tip radius/region increases [19]. During the 3D printing process, a significant phenomenon called resin flow occurs. This phenomenon is present at photopolymerization methods and it is generated from up and down movements of printing platform along the Z-axis. In the current case it is believed that the resin flow phenomenon directs resin material on the surfaces of microchannel walls. Due to the upcoming polymerization cycle, excess resin material is polymerized, and microchannel obstruction is caused.

As the results demonstrated, resin flow depends strongly on geometry. Beveled configuration prevents microchannel clogging due to their inclined surface, which results to a widened ellipsoid ending. 
It also seems that microchannel formation is not affected by HMN geometry itself, in the sense that external wall configuration (tetrahedron, hexahedron et cetera) has no impact on clogging or not of microchannels.

The most critical parameter for HMN fabrication is the printing angle, which is responsible for resin flow effect. It is believed that $0^{\circ},+45^{\circ}, 90^{\circ}$ angles favour resin displacement across HMN channels resulting in clogging. On the contrary, $-45^{\circ}$ minimizes resin flow effect on microchannel surface preventing thus from clogging (beveled geometry). Resin flow minimization at $-45^{\circ}$ can be attributed to the fact that the inclined surface of the HMNs is almost vertical to $\mathrm{Z}$ axis, as demonstrated in Figure 3(a). The influence of resin flow by $3 \mathrm{D}$ printing angle has been also reported in a previous work from our group [20]. Briefly, when a constructed surface of the printlet is parallel to the building platform, then resin flow phenomenon is minimized at the maximum degree (Figure 4). A typical image of the optimal 3D printed HMN array is shown in Figure 3(b).

The geometries and the 3D printing angles along with the corresponding result of microchannel formation are presented in Table 1.
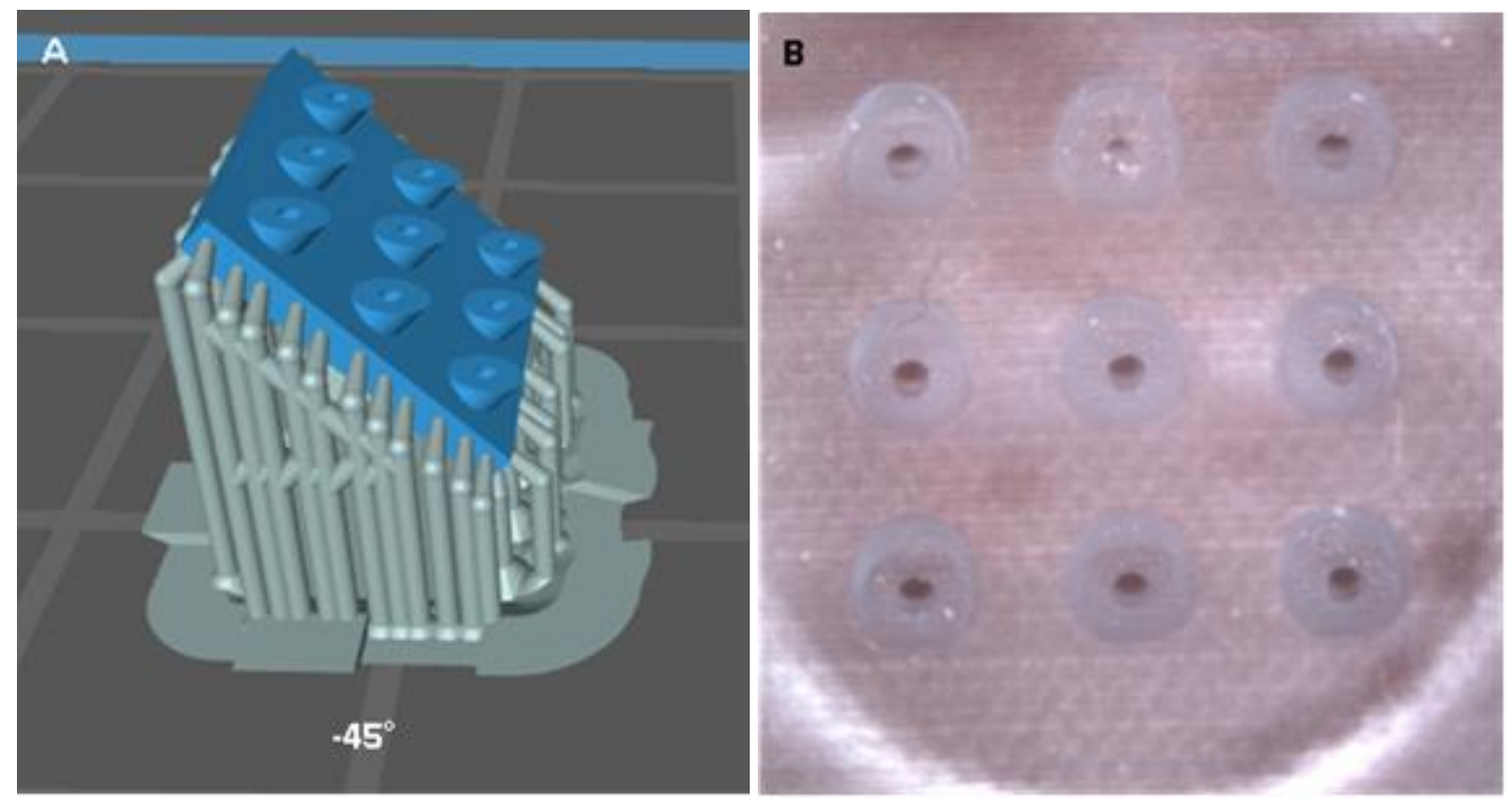

Fig. 3. (a) Beveled HMN patch at $-45^{\circ}$. Design derived from ChiTuBox slicing software. Inclined surface of the HMNs is almost vertical to $\mathrm{Z}$ axis. (b) Top view of beveled 3D-printed HMNs at $-45^{\circ}$

Table 1. Summarized results of successful or not microchannel formation. All 3D-printings included 10s curing time, $400 \mu \mathrm{m} / \mathrm{s}$ lifting speed and $30 \mu \mathrm{m}$ layer height

\begin{tabular}{|c|c|c|}
\hline Geometry & $\begin{array}{c}\text { Non-clogged } \\
\text { microchannel }\end{array}$ & 3D Printing Angles \\
\hline beveled & Yes & $-45^{\circ}$ \\
\hline beveled & No & $0^{\circ}, 90^{\circ},+45^{\circ}$ \\
\hline hexagon & No & $0^{\circ}, 90^{\circ},+45^{\circ},-45^{\circ}$ \\
\hline square pyramid & No & $0^{\circ}, 90^{\circ},+45^{\circ},-45^{\circ}$ \\
\hline
\end{tabular}




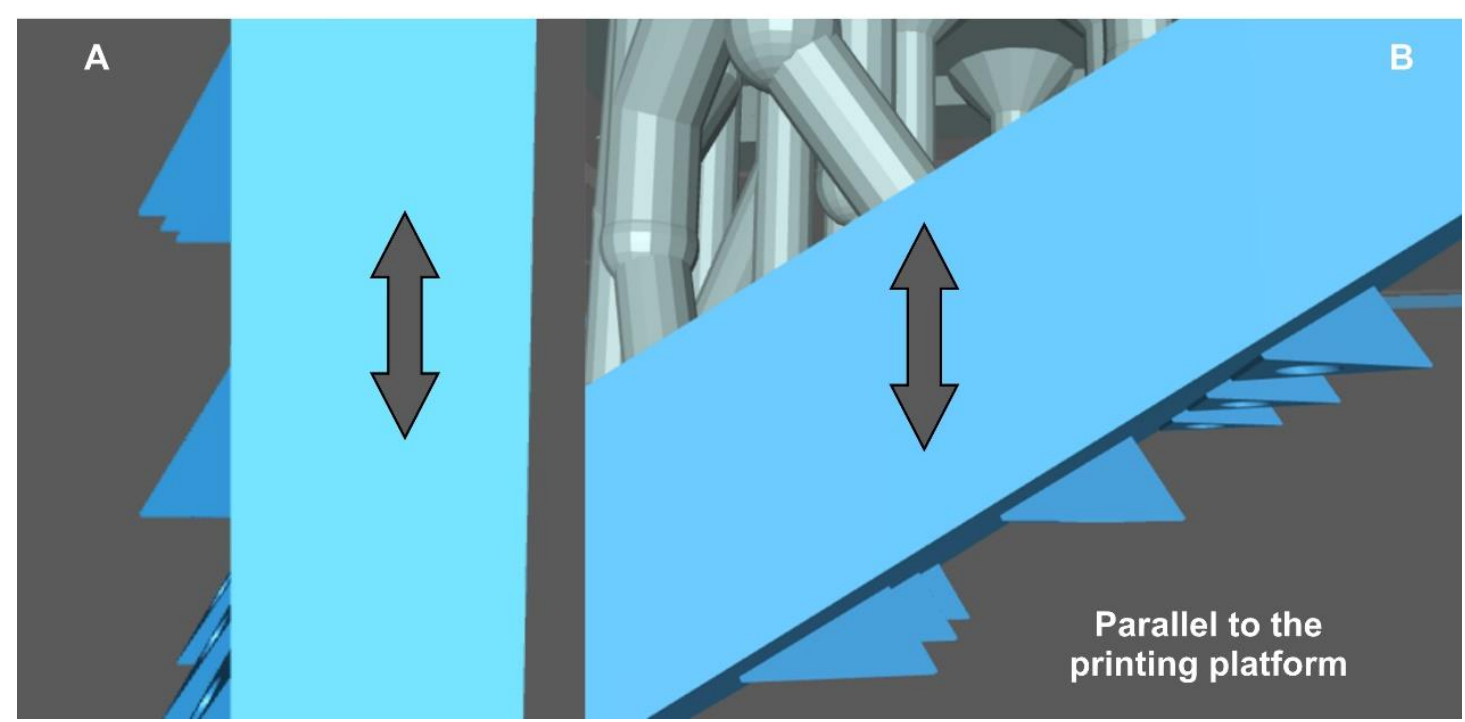

Fig. 4. Schematics derived from ChiTuBox slicing software. At the left, HMNs are positioned at $+90^{\circ}(\mathrm{A})$. At the right, inclined surface of HMNs is parallel to the printing platform (B). In case (B), microchannel is considered "protected" from resin flow phenomenon

Lastly, resin flow may be the reason for slightly coarser tip area at $-45^{\circ}$. This could be explained by the fact that at the rest $3 \mathrm{D}$ printing angles $\left(0^{\circ}, 90^{\circ}\right.$, $+45^{\circ}$ ), the resin flow phenomenon causes microchannel obstruction and this restrains resin quantity flowing on the tip.

It must be noted that all 3D-printings were carried out at a constant lifting speed. It is certain that increasing or decreasing lifting speed changes all rheological phenomena, which in turn may influence the final result of a 3D-printing. Microchannel formation under different 3D printing angles may also be affected if microchannel dimensions are modified. It should be noted that microchannel dimensions can vary due to the accuracy each 3Dprinter offers, too. If printing accuracy is lowered, more than the wished layers may be polymerized enhancing the possibility of microchannel narrowing. Another factor affecting resin flow phenomenon is the resin itself. Each resin has specific physical characteristics, one of which is viscosity. Viscosity affects by definition the extent by which resin flow occurs.

Considering all factors that must be met for a successful 3D printing with VPMs, one could argue about the suitability of the proposed method for HMN fabrication. Yet, all issues emerged were successfully solved and feedback from the current research could be used for future HMN fabrication. In addition, AM methods offer more than convenience and flexibility during fabrication procedure; the simple equipment required has the dynamic to transform the current trends in the market. To be more specific, product manufacturing nowadays is based on a central oriented model, where one or few large production units (factories) supply all the interested consumers across the world. This model prerequisites transportation of products which in turn increases the final cost. On the contrary, production through AM methods require a 3D-printer, a computer and a designing software. This quite simple equipment can be used to establish small production units even at the remotest regions of the world diminishing the need for transportation and thus the final cost. Consequently, products can be more accessible to developing countries and low income individuals. Finally, products and in this case HMNs, can be manufactured on demand for a specific patient after a short period of time. [21,22]

\section{CONCLUSIONS}

In this work, several geometries of HMNs were designed and fabricated by means of $3 \mathrm{D}$ printing technology in an attempt to investigate the best possible configuration. Optimum beveled HMNs of $900 \mu \mathrm{m}$ height and relatively low tip area (around $4500 \mu \mathrm{m}^{2}$ ) were fabricated using LCD vat polymerization method under the following parameters: i) curing time $10 \mathrm{~s}$, ii) $3 \mathrm{D}$ printing angle $-45^{\circ}$ and iii) layer resolution $30 \mu \mathrm{m}$. Further research on parameters affecting microchannel formation could take into consideration lifting speed of the building platform, dimensions of the microchannels and use of different resin materials emphasizing on viscosity characteristic. Yet, due to the purpose of 
our research, future studies will include flow ability studies, mechanical testing and permeability studies of insulin across human skin in vitro.

\section{ACKNOWLEDGEMENTS}

This research is co-financed by Greece and the European Union (European Social Fund- ESF) through the Operational Programme «Human Resources Development, Education and Lifelong Learning 2014-2020» in the context of the project "Fabrication of 3D printed microneedles for transdermal personalized delivery of insulin" (MIS 5047927).

\section{REFERENCES}

1. McMurtry M, Noel M, Taddio A, Antony M, Asmundson G, Riddell R-P, Chambers C, Shah V, $\mathrm{N}$, Rogers J, Bucci L, Mousmanis P, Lang E, Halperin S, Bowles S, Halpert C, Ipp M, Rieder M, Robson K, Uleryk E, Dubey V, Hanrahan A, Lockett D, Scott J and Bleeker V 2015 Clin. J. Pain 31(10) 109-123.

2. Wright S, Yelland M, Heathcote K, Ng S-K and Wright G 2009 Austr. Fam. Phys. 38(3) 172-176.

3. Hultström M, Roxhed N and Nordquist L $2014 \mathrm{~J}$. of Diab. Sci. \& Techn. 8(3), 453-457.

4. R, Patel M, Maahs D and Shah V 2016 Int. J. Pharm. Inv. 6(1):1.

5. Kim Y-C, Park J-H and Prausnitz M $2012 A d v$. Drug Del. Rev. 64(14) 1547-1568.

6. Zhang P, Dalton C and Jullien G 2009 Microsys. Techn. 15(7) 1073-1082.

7. Jamroz W, Kurek M, Lyszczarz E, Brniak W and Jachowicz R 2017. Act. Pol. Pharm. 74(3) 753-763.

8. Yeung C, Chen S, King B, Lin H, King K, Akhtar F, Diaz G, Wang B, Zhu J, Sun W, Khademhosseini A and Emaminejad S 2019 Biomicrofl. 13(6).

9. Farias C, Lyman R, Hemingway C, Chau H, Mahacek A, Bouzos E and Mobed-Miremadi M 2018 Bioeng. 5(3).

10. Economidou $\mathrm{S} \mathrm{N}$, Uddin $\mathrm{M} \mathrm{J}$, Marques $\mathrm{M} \mathrm{J}$, Douroumis D, Sow W T, Li H, Reid A, Windmill J FC and Podoleanu A 2021 Add. Manuf. 38101815.

11. Gittard S, Ovsianikov A, Monteiro-Riviere N, Lusk J, Morel P, Minghetti P, Lenardi C, Chichkov B, and Narayan R 2009 J. Diab. Sci. Techn. 3(2) 304-311.

12. Szeto B, Aksit A, Valentini C, Yu M, Werth EG, Goeta S, Tang C, Brown LM,

Olson ES, Kysar JW and Lalwani AK 2021 Hear. Res. 400.

13. Ovsianikov A, Chichkov B, Mente P, MonteiroRiviere N-A, Doraiswamy A, and
Narayan R-J 2007 Int. J. Appl. Ceram. Technol. 4 2229.

14. Doraiswamy A, Jin A, Narayan R J, Mageswaran P, Mente P, Modi R, Auyeung R, Chrisey D B, Ovsianikov A and Chichkov B 2006 Act. Biomater. 2 267-275.

15. Trautmann A, Roth G L, Nujiqi B, Walther T and Hellmann R 2019 Microsyst. Nanoeng. 5.

16. Luzuriaga M, Berry D, Reagan J, Smaldone R and Gassensmith J 2018 Lab. Chip 18(8) 12231230 .

17. Derakhshandeh H, Aghabaglou F, McCarthy A, Mostafavi A, Wiseman C, Bonick Z, Ghanavati I, Harris S, Kreikemeier-Bower C, Masoud S, Basri M, Rosenbohm J, Yang R, Mostafalu P, Orgill D and Tamayol A 2020 Adv. Funct. Mat. 30(13) 1905544.

18. Gieseke M, Senz V, Vehse M, Fiedler S, Irsig R, Hustedt M, Sternberg K, Nölke C, Kaierle S, Wesling V, Tiggesbäumker J, Meiwes-Broer K H, Seitz H, Schmitz K P and Haferkamp H 2012 Biomed. Techn. 57 398-401.

19. Xenikakis I, Tzimtzimis $\mathrm{M}$, Tsongas $\mathrm{K}$, Andreadis K, Demiri E, Tzetzis D and Fatouros D 2019 Eur. J. Pharm. Sci. 137104976.

20. Xenikakis I, Tsongas K, Tzimtzimis M, Zacharis K, Theodoroula N, Kalogianni E, Demiri E, Vizirianakis I, Tzetzis D and Fatouros D 2021 Int. J. Pharm. 597120303.

21. Thomas D 2016 Int. J. of Adv. Man. Techn. 85(5-8), 1857-1876.

22. Alomari M, Mohamed F H, Basit A W and Gaisford S 2015 Int. J. of Pharm. 494(2) 568-577.

Received: March 26, 2021 / Accepted: December 20, 2021 / Paper available online: December 25, 2021 (C) International Journal of Modern Manufacturing Technologies 